\title{
ACTA BIOETHICA: UNA DÉCADA DE HISTORIA
}

\author{
Fernando Lolas Stepke*
}

Resumen: Se presenta una síntesis histórica del origen y evolución de Acta Bioethica (ISSN 0717-5906), destacando los temas abordados en sus primeros diez años y el origen geográfico de los artículos publicados. Indexada en SciELO, Science Citation Index, Latindex y otras bases de datos internacionales, la revista se ha convertido en referencia obligada para los estudiosos. Sus artículos, en inglés, español y portugués, se citan cada vez con mayor frecuencia. El Centro Interdisciplinario de Estudios en Bioética (CIEB) de la Universidad de Chile, que la editó durante la vigencia del consorcio con el Programa de Bioética OPS/OMS, continúa incrementando su presencia en los medios especializados.

Palabras clave: publicación bioética, Acta Bioethica, historia bioética

\section{ACTA BIOETHICA: A DECADE OF HISTORY}

Abstract: A historical account of origin and evolution of Acta Bioethica (ISSN 0717-5906) is presented, emphasizing the topics addressed and the geographical origin of its authors during its first ten years of existence. Indexed in SciELO, Science Citation Index, Latindex and other bibliographic databases, the journal has become standard reference for scholars. Its papers, in English, Spanish and Portuguese, are cited with increasingly higher frequency. The Centro Interdisciplinario de Estudios en Bioética (CIEB), at the University of Chile, which edited it during the time of the consortium with The PAHO/WHO Bioethics Program, continues increasing its presence in specialized circles.

Key words: bioethics publication, Acta Bioethica, bioethics history

\section{ACTA BIOETHICA: UMA DÉCADA DE HISTÓRIA}

Resumo: Apresenta-se uma síntese histórica da origem e evolução da Acta Bioethica (ISSN 0717-5906), destacando-se os temas abordados nos seus primeiros dez anos e a origem geográfica dos artigos publicados. Indexada em SciELO, Science Citation Index, Latindex e outras bases de dados internacionais, a revista se tornou uma referência obrigatória para os estudiosos. Seus artigos em inglês, espanhol e português são citados cada vez mais e com maior frequência. O Centro Interdisciplinar de Estudos em Bioética (CIEB) da Universidade do Chile, que a editou durante a vigência do consórcio com o Programa de Bioética OPS/OMS, continua incrementando a sua presença nos meios especializados.

Palavras-chave: publicação bioética, Acta Bioethica, história bioética

* Centro Interdisciplinario de Estudios en Bioética, Universidad de Chile, Chile. Grant TW.0056

Correspondencia: flolas@uchile.cl 


\section{Contexto e introducción}

Este artículo expone lo que ha sido Acta Bioethica en sus diez ańos de existencia, sus aportes y su futuro. Su creación (el año 2000) obedeció a tres propósitos. Primero, fomentar la producción intelectual en temas de ética médica, ética de la investigación, ecoética y disciplinas relacionadas en el ámbito latinoamericano. En segundo lugar, propender al cultivo solvente de una disciplina intelectual que permitiera, tras algunos ańos, superar las evidentes superficialidades e improvisaciones que caracterizaban y caracterizan a lo que en el continente americano muchos llaman "bioética". Finalmente, proporcionar un foro de intercambio de experiencias que pudiera entrar al concierto internacional con respetabilidad.

La revista debía cumplir un papel en la "agenda bioética" que habíamos diseñado desde el Centro Interdisciplinario de Estudios en Bioética (CIEB) de la Universidad de Chile, especialmente durante el período que durara su asociación con la Organización Panamericana de la Salud (OPS) para la operación conjunta del Programa de Bioética iniciado en 1994, a instancias de la Universidad y del Gobierno de Chile, y concluido el año 2010(1,2,3). Durante el trabajo en consorcio, la revista recibió apoyo para su edición del Programa de Bioética, pero se mantuvo, como las monografías que la complementaron, bajo la supervisión del CIEB. La colección completa, junto con otras publicaciones relevantes, pueden consultarse en www.bioetica.uchile. $\mathrm{cl}$ y en el sitio de la revista www.actabioethica.cl. Este último se desarrolló, junto con otros perfeccionamientos editoriales, gracias al apoyo del Fondo para Publicaciones Científicas de CONICYT Chile con aportes obtenidos por concurso en el año 2008.

La experiencia con publicaciones en Latinoamérica, Europa y Estados Unidos indicaba la necesidad de crear un comité editorial amplio y dispuesto a colaborar activamente en la revisión de manuscritos y cautelar la calidad de lo publicado. El primer intento de crear tal comité en el medio chileno fracasó. Era muy numeroso, los convocados no entendieron el cometido y se perdió un año en reuniones que apuntaban a jerarquizar y formalizar algo que aún no existía. La experiencia reiteró otras semejantes y fortaleció la impresión de que esta área (bioética) no tenía entonces una identidad suficiente como para establecer estándares rigurosos.

Cuando el año 2000 finalmente apareció el primer número, la revista ofrecía el pluralismo como una fortaleza y prometía publicar números monográficos que abordaran un tema a cargo de editores invitados que contribuirían a seleccionar los artículos, sin que ello significara reducir la consulta a pares evaluadores externos. La revista recibió su International Standard Serial Number y empezó a publicarse regularmente en entregas semestrales(4). Para su numeración en términos de años, se consideró la continuidad con una revista de divulgación previa, los Cuadernos del Programa Regional de Bioética, que carecía de carácter académico.

\section{Las temáticas y los autores}

Los anexos, preparados por el actual editor Álvaro Quezada, indican los temas abordados en cada número, la procedencia de los trabajos publicados y los nombres de los editores invitados. La mayoría de los números monográficos se prepara a solicitud de los editores invitados, lo cual no es óbice para una revisión adicional por pares externos. La existencia de un tema central no excluye la posibilidad de publicar artículos de otra temática. De hecho, la cantidad y calidad de artículos remitidos en forma espontánea creció en forma notable después que la revista fuera invitada a integrar SciELO (Scientific Electronic Library on Line) y más aún con la incorporación a Science Citation Index (Thomson Reuters). Estos artículos, denominados "miscelánea", suelen agregarse a los del tema principal según disponibilidad de espacio después de su aceptación formal. Para publicar en Acta Bioethica, los autores deben indicar sus conflictos de interés y asegurar originalidad y autoría responsable.

Cada artículo se acompaña de resúmenes en inglés, portugués y español, los tres idiomas de publicación, y palabras-clave que permiten indexación.

La revista publica también recensiones de libros y comentarios de publicaciones en otros idiomas. Tales referencias permiten acceder a textos no habituales. Se cumple así una función difusora y didáctica, especialmente útil en el medio latinoamericano.

\section{Etapas de desarrollo}

En sus diez años de existencia, Acta Bioethica ha recibido el apoyo de numerosos expertos y académicos y ha publicado en español, portugués e inglés. En los últimos años ha diversificado su consejo asesor y sus colaboradores editoriales, incorporando como editores asociados a académicos de Estados Unidos y Brasil. 
La contribución del Programa de Bioética OPS/OMS contribuyó a posicionar la publicación entre los funcionarios de esa organización internacional. Su índice de impacto ha crecido en los últimos años.

El futuro de la publicación considerará un uso intenso de la plataforma electrónica y el open access. Se espera mantener un intercambio fluido y permanente con los autores estimulando los comentarios y las cartas al director. La renovación del comité editorial seguirá realizándose cada dos años, sin perjuicio de que algunos de sus miembros puedan ser invitados a continuar en funciones por más de un período.

\section{Referencias}

1. Lolas F. El discurso bioético. Una anécdota personal. Quirón (La Plata) 1994; 25(4): 28-30.

2. Lolas F. El discurso bioético: una anécdota personal. Vida Médica (Santiago de Chile) 1994; 46 (3): 22-24.

3. Lolas F. Bioética en la Universidad de Chile. Anales de la Universidad de Chile 1998; Sexta Serie, 8: 13-18.

4. Lolas F. Editorial. Acta Bioethica 2000; 6(1): 5-6.

\section{Anexo I}

Títulos de Acta Bioethica publicados hasta la fecha

\begin{tabular}{|c|c|c|l|}
\hline Año & Vol & No & Título \\
\hline 2000 & 6 & 1 & Cuidados paliativos y bioética \\
\hline 2000 & 6 & 2 & Perspectivas bioéticas y publicaciones biomédicas \\
\hline 2001 & 7 & 1 & Envejecimiento y calidad de vida \\
\hline 2001 & 7 & 2 & Nocividad ambiental y alimentaria \\
\hline 2002 & 8 & 1 & Debate ético y ciencias sociales \\
\hline 2002 & 8 & 2 & Derecho y bioética \\
\hline 2003 & 9 & 1 & Biotecnología y ética \\
\hline 2003 & 9 & 2 & Bioética y salud pública \\
\hline 2004 & 10 & 1 & Bioética e investigación en seres humanos \\
\hline 2004 & 10 & 2 & Bioética y genómica \\
\hline 2005 & 11 & 1 & Politicas públicas \\
\hline 2005 & 11 & 2 & Tecnologías de la información \\
\hline 2006 & 12 & 1 & Bioética y odontoestomatología \\
\hline 2006 & 12 & 2 & Bioética y género \\
\hline 2007 & 13 & 1 & Experimentación con animales y bioética \\
\hline 2007 & 13 & 2 & Bioética y salud reproductiva \\
\hline 2008 & 14 & 1 & Enseñanza de la bioética \\
\hline 2008 & 14 & 2 & Experiencias en bioética \\
\hline 2009 & 15 & 1 & Fundamentación de la bioética \\
\hline 2009 & 15 & 2 & Perspectivas en salud mental \\
\hline 2010 & 16 & 1 & Religión y bioética \\
\hline
\end{tabular}

\section{Anexo 2}

Editores invitados

\begin{tabular}{|c|}
\hline Lorenzo Agar Corbinos \\
\hline Alexandre Bota Arqué \\
\hline Marcela Ferrer Lues \\
\hline Kenneth W. Goodman \\
\hline Francisco León Correa \\
\hline Javier Luna Orosco \\
\hline Adelio Misseroni Raddatz \\
\hline Mauricio Olavarría Gambi \\
\hline Luis Risco Neira \\
\hline Eduardo Rodríguez Yunta \\
\hline Paulina Taboada Rodríguez \\
\hline Carolina Valdebenito Herrera \\
\hline
\end{tabular}




\section{Anexo 3}

Artículos de Acta Bioethica según país de autores

\begin{tabular}{|c|c|c|c|c|c|c|c|c|c|c|c|c|c|c|c|c|c|c|c|c|c|c|}
\hline & 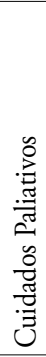 & 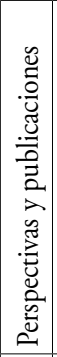 & 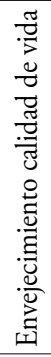 & 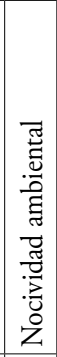 & 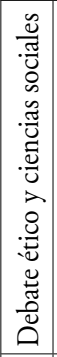 & 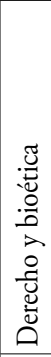 & 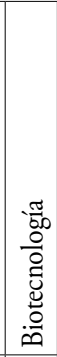 & 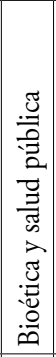 & 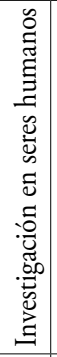 & 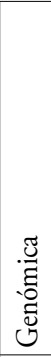 & 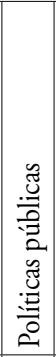 & 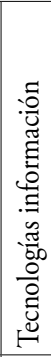 & 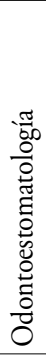 & : & 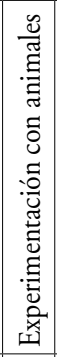 & 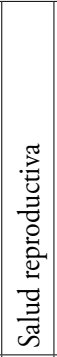 & 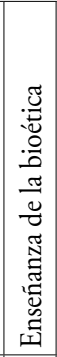 & 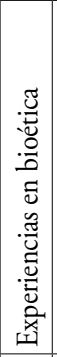 & 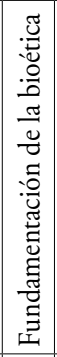 & 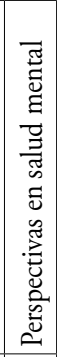 & 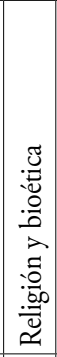 & $\stackrel{\pi}{0}$ \\
\hline Alemania & & & & & 1 & & & & & & 1 & & & & & & & & & & & 2 \\
\hline Argentina & 2 & 3 & 1 & 1 & 1 & 2 & 2 & 1 & 1 & 1 & 3 & 3 & 4 & 1 & & 3 & 2 & & 2 & & & 33 \\
\hline Australia & & & & 1 & & & & & & & & & & & & & & & & & & 1 \\
\hline Bolivia & & & & & & & & & & & & & & & & & & & & & 1 & 1 \\
\hline Bélgica & & & 1 & & & & & & & 1 & & & & & & & & & & & & 2 \\
\hline Brasil & & 1 & & 2 & 1 & 2 & 2 & & & & & & & 1 & 1 & & & 1 & 2 & 1 & 2 & 16 \\
\hline Canadá & & & & & & & & 2 & & & & 1 & & & & & & & & 3 & & 6 \\
\hline Chile & 6 & 5 & 9 & 6 & 4 & 2 & 2 & 3 & 3 & 5 & 2 & 2 & 4 & 7 & 4 & 7 & 4 & 5 & 4 & 6 & 4 & 94 \\
\hline Colombia & & & & & 1 & & & & & & & & 3 & 1 & 1 & 1 & & & & & & 7 \\
\hline Costa Rica & & & & & & & & & & 1 & & & & & & & & & & & & 1 \\
\hline Cuba & & & & & & & & & & & & & & & 1 & & & 1 & & & & 2 \\
\hline Ecuador & & & & & & & & & & & & & & 1 & & & & & & & & 1 \\
\hline España & 2 & 1 & & 1 & 1 & 3 & 3 & & 1 & 1 & 1 & & & & 3 & 1 & 1 & & 2 & & 1 & 22 \\
\hline Estados Unidos & & & 2 & & 1 & & 1 & 2 & 3 & 1 & 1 & 3 & 1 & & 1 & & 1 & & & & & 17 \\
\hline Francia & & 1 & & & 1 & & & & & & & & & & & & & & & & & 2 \\
\hline India & & & & & & & & & & & & & & & & & & & & & 1 & 1 \\
\hline Inglaterra & 1 & & & & & & & 1 & 2 & & & & & & & & & & & & & 4 \\
\hline Italia & & & & 1 & & 2 & & & & & & & & & 3 & & 1 & & 1 & 1 & & 9 \\
\hline México & & & & & & & 1 & 1 & & & & 1 & 2 & 1 & 1 & 1 & & 4 & 1 & & 1 & 14 \\
\hline Nicaragua & & & & & & & & & & & & & & 1 & & & 1 & 1 & & 1 & & 4 \\
\hline Panamá & & & & & & & & & & 1 & & & & & 1 & & & & & & & 2 \\
\hline Perú & & & & & & & & & & 1 & & & & 1 & & & 2 & 1 & & & & 5 \\
\hline Polonia & & & & & & & & & & & 1 & & & & & & & & & & & 1 \\
\hline Puerto Rico & & & & & 1 & & & & & & & & & & & & & & 1 & 1 & & 3 \\
\hline República Dominicana & & & & & & & & & & & & & & & & & & & & & 1 & 1 \\
\hline Suiza & & & & 1 & & & & & & & & & & & & & & & & & & 1 \\
\hline Venezuela & & & & & & & & & & & & & & & & & 2 & & & & & 2 \\
\hline No artículos & 11 & 11 & 13 & 13 & 12 & 11 & 11 & 10 & 10 & 12 & 9 & 10 & 14 & 14 & 16 & 13 & 14 & 13 & 13 & 13 & 11 & 254 \\
\hline
\end{tabular}

Recibido: 1 de octubre de 2010

Aceptado: 2 de octubre de 2010 